(C) 2015 IEEE. Personal use of this material is permitted. Permission from IEEE must be obtained for all other uses, in any current or future media, including reprinting/republishing this material for advertising or promotional purposes, creating new collective works, for resale or redistribution to servers or lists, or reuse of any copyrighted component of this work in other works. 


\section{A Hybrid Short-term Solar Power Forecasting Tool}

\author{
J.M. Filipe, R.J. Bessa, J. Sumaili \\ Center for Power and Energy Systems \\ INESC TEC \\ Porto, Portugal \\ jorge.m.filipe@inesctec.pt, ricardo.j.bessa@inesctec.pt, \\ jean.sumaili@inesctec.pt
}

\author{
R. Tomé ${ }^{1,2}$, J.N. Sousa ${ }^{1}$ \\ ${ }^{1}$ Prewind Lda. \\ Porto, Portugal \\ 2 IDL - Instituto Dom Luiz \\ Lisboa, Portugal \\ ricardo.tome@prewind.eu,joao.sousa@prewind.eu
}

\begin{abstract}
Photovoltaic (PV) solar power capacity is growing in several countries, either concentrated in medium/large size solar parks or distributed by the medium and low voltage grid. Solar power forecasting is a key input for supporting grid management, participation in the electricity market and maintenance planning. This paper proposes a new forecasting system that is a hybrid of different models, such as electrical and statistical models, as well as different Numerical Weather Prediction (NWP) sources. The time horizon is 48 hours ahead. The proposed model was operationalized and tested in a real PV installation located in North Portugal with $16 \mathrm{~kW}$.
\end{abstract}

Keywords; solar power; forecasting; electrical model; statistical model; numerical weather predictions.

\section{INTRODUCTION}

The economics of photovoltaic (PV) solar power are presently very attractive to final electricity consumers since PV generation is reaching grid parity in comparison to retailing tariffs [1]. Besides, several European countries are designing a regulatory framework to support selfconsumption at the building level [2], the "prosumer" concept. The investment in medium/large scale solar plants is also growing and their integration in the wholesale electricity market is expected.

In this context, short-term solar power forecasting is an important service for both system operators and PV installations owners/operators. It can be used in grid management functions, such as setting the operating reserve requirements [3] and voltage control [4], as well as to define electricity market offers. The time horizon of interest for these functions is between 48 hours and one week ahead, frequently called short-term horizon [5].

The literature about solar power forecasting is rich in machine learning methods applied to extrapolate solar power from Numerical Weather Predictions (NWP). For instance, Fernandez-Jimenez et al. [6] used NWP as input in several machine learning algorithms (i.e., Auto-Regressive Integrated Moving Average - ARIMA, k-nearest neighbours - $\mathrm{kNN}$, neural networks - NN, and adaptive neuro-fuzzy models) to produce solar power forecasts for the next 39 hours. Zamo et al. [7] compared several regression algorithms (e.g., random forests, boosting, support vector machines - SVM) that take NWP as input to produce solar power forecasts. Chen et al. [8] combined a self-organized map (SOM), which classifies the local weather type of 24 hours-ahead NWP, with a neural network that converts the NWP information into solar power. Bacher et al. [9] proposed an autoregressive model with exogenous inputs (i.e., NWP).

For the very short-term horizon (e.g., up to ten hours ahead), the state of the art consists in ARIMA models with past observations of the same time series as input [10]. Bessa et al. [11] developed a vector autoregressive model (VAR) that combines information from distributed smart meters (associated to micro-generation) and remote terminal units from a smart grid in order to improve the forecast skill. A more detailed literature review can be found in [12].

These models should be seen as components in a complete forecasting system, which should include different types of models and cover multiple time horizons. This papers aims to describe the architecture and algorithms of a solar power forecasting tool. The proposed architecture has two innovative features:

a. Combines an electrical model (described in [13]) of the PV system and a statistical model (gradient boosting technique [14]) that converts NWP into solar power for the short-term time horizon;

b. Different NWP models are combined with information from past observations of the time series.

The forecast time horizon is 48 hours ahead and the final output are probabilistic forecasts (i.e., set of quantiles). Moreover, a mesoscale NWP model was parameterized for Portugal in order to produce high resolution global horizontal and direct irradiance predictions.

It is important to stress that the electrical based model provides a better characterization and interpretation of the physical system and does not need to know in advance the PV plants characteristics as they are estimated by a metaheuristic optimization algorithm. Statistical models are combined with this module in order to improve the overall forecasting skill.

The paper is organized as follows: section II describes the architecture of the forecasting tool; section III describes the NWP model and corresponding setup for Portugal; sections IV presents the chain of algorithms to produce point

This work was made in the framework of the PREVSOL project ("NORTE-07-0202-FEDER-038588") co-financed by the North Portugal Regional Operational Programme (ON.2 - O Novo Norte), under the National Strategic Reference Framework (NSRF), through the European Regional Development Fund (ERDF). It was also co-financed by the BEST CASE project ("NORTE-07-0124-FEDER-000056") financed by the ON.2 - O Novo Norte, under the NSRF, through the ERDF, and by national funds, through Fundação para a Ciência e a Tecnologia (FCT). 
forecasts; section $\mathrm{V}$ describes the probabilistic forecasting algorithm; the test case results are presented in section VI; section VII presents the conclusions.

\section{ARCHITECTURE OF THE FORECASTING TOOL}

The architecture of the solar power forecasting tool is depicted in Fig. 1. It consists of a hybrid of statistical and physical models (e.g., electrical and NWP model).

For the very short-term horizon (i.e., up to 10 hoursahead), an autoregressive model (AR) that takes as input past observations from the time series is used. For the shortterm horizon, the information comes from a set of NWP models that feed two different types of algorithms (i.e., electrical and statistical models) that convert solar irradiance into power.

The solar power forecasts generated by the AR model and by the short-term algorithms created with different NWP are linearly combined (i.e., linear pool) in order to produce a single point forecast.

Finally, a local quantile regression model produces a set of quantile forecasts (e.g., probabilistic forecasts) using as input the point forecast generated by the linear pool. This final output can be used to derive electricity market offers or to plan maintenance operations.

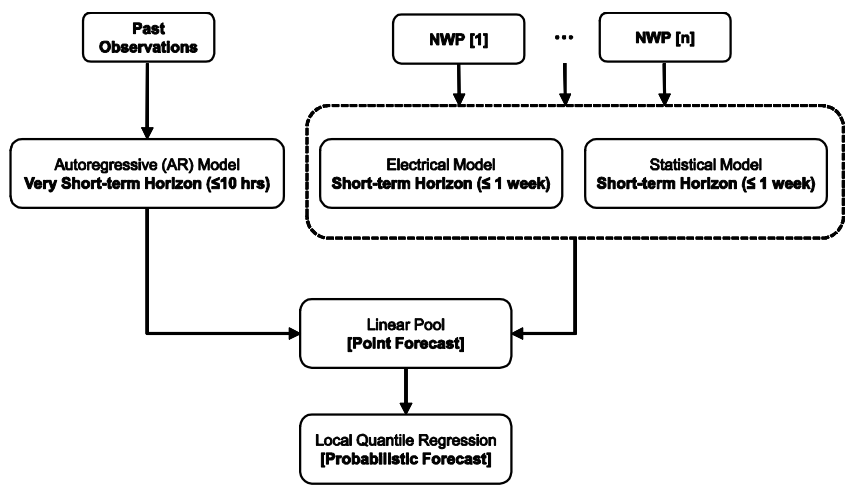

Figure 1. Architecture of the solar power forecasting tool.

\section{PARAMETRIZATION OF THE NWP MODEL}

The Weather Research and Forecasting (WRF) [15] modeling project is a community effort intended to develop a next-generation mesoscale forecast model and dataassimilation system that will advance both the understanding and prediction of mesoscale weather, and accelerate the transfer of research advances into operations.

The WRF model is well suited for a wide range of applications, from idealized research simulations to operational forecasting, and has the flexibility to accommodate future enhancements. Although the model is designed to improve forecast accuracy across scales ranging from cloud to synoptic, the emphasis on horizontal grid resolutions of 1-10 km makes WRF particularly well suited for newly emerging numerical weather prediction applications in the non-hydrostatic regime.

The weather forecasts used here, come from a Prewind operational weather forecast model, which is configured with two nested domains using one-way nesting, the outer domain with $16 \mathrm{~km}$ horizontal resolution and the inner domain with $4 \mathrm{~km}$ horizontal resolution.

The model radiation package follows the Rapid Radiation Transfer Model (RRTM) [16]. The RRTM package aims to calculate shortwave and longwave fluxes that simulate the absorption of radiation by water vapor, ozone, etc., and simulate phenomena's like reflection of radiation in the Earth surface and clouds, scattering of radiation due to the presence of aerosols and clouds. This provides the necessary output to calculate all the necessary variables to solar power forecasting.

\section{POINT FORECAST MODELS}

\section{A. Autoregressive Model}

The very short-term model covers a time horizon up to 10 hours ahead and it is based on the autoregressive (AR) process [17]. The AR model for time interval $t$ can be interpreted as a regression on past observations (or lags) of the time series. For one hour-ahead forecast, the AR model is the following:

$$
\hat{p}_{t+1 \mid t}^{n}=\beta_{0}+\beta_{1} \cdot p_{t}^{n}+\beta_{2} \cdot p_{t-1}^{n}+\cdots+\beta_{l} \cdot p_{t-l}^{n}+\varepsilon_{t+1 t}
$$

where $\beta$ are the model's coefficients, $l$ the order of the AR model and $\varepsilon_{t+1 \mid t}$ is a white noise process with zero mean and constant variance. Note that $p^{n}$ is the solar power time series normalized in order to remove the seasonal component with the weighted quantile regression model (or clear-sky model) described in [9].

The coefficients of the model in Eq. 1 are estimated with the Recursive Least Squares (RLS) method with forgetting factor, as described in [17].

Since this model is unable to completely remove the seasonal component of the model, a diurnal term corresponding to lag $t-23$ (for hourly time series) is included. Following the results in [9][11], the lags $t$ and $t-1$ are also introduced in the model.

In this paper, multi-step ahead forecasts are produced. Therefore, a different model is fitted for each lead-time, ranging between 1 and 10 hours ahead.

\section{B. Electrical Model}

The electrical model uses NWP has an input and its parameters are found by an optimization process aimed at reducing the forecast error of the training dataset. This model exploits meaningful parameters in opposition with black-box, such as neural network, and presents the advantage of including user knowledge for the definition of the limits of the parameters.

The model aims at transforming NWP into PV outputs. For doing so, it is necessary to separate the forecasted global irradiance into three different components to estimate the global irradiance on a tilted surface. Then it can be converted into PV power output by considering the influence of cell temperature and inverter losses according to the ambient conditions [18].

\section{1) Tilted Plane Global Irradiance}

The three components of the incident global irradiance are [19]: (i) the beam component from direct irradiation of the tilted surface $G_{b, \beta}$, (ii) the diffuse component $G_{d, \beta}$ and (iii) the reflected component that quantifies the radiation reflected from the ground to the tilted surface $G_{r, \beta}$, i.e.,

$$
G_{g, \beta}=G_{b, \beta}+G_{d, \beta}+G_{r, \beta}
$$


The calculation of the tilted direct component $G_{b, \beta}$ is purely geometrical

$$
G_{b, \beta}=B_{n} \cos \theta_{i}=\frac{B}{\cos \theta_{z}} \cos \theta_{i}
$$

where

$$
\theta_{i}=\cos ^{-1}\left[\cos \theta_{z} \cos \beta+\sin \beta \sin \theta_{z} \cos \left(\theta_{a}-\varphi_{a}\right)\right]
$$

is the incidence angle [20], $B_{n}$ the direct beam referred in NWP as the Direct Normal Irradiance (DNI), $B$ the Direct Horizontal Irradiance (DHI), $\beta$ the tilted angle, $\theta_{z}$ the sun zenith angle, $\theta_{a}$ the sun azimuth angle and $\varphi_{a}$ the surface azimuth angle.

To estimate the diffuse component, one can use the model developed at Sandia National Laboratories in order to determine the diffuse irradiance from the sky on a tilted surface using the surface tilt angle, diffuse horizontal irradiance, global horizontal irradiance, and sun zenith angle [21].

$$
G_{d, \beta}=B\left(\frac{1+\cos \beta}{2}\right)+G H I \cdot\left(0.012 \cdot \theta_{z}-0.04\right) \cdot\left(\frac{1-\cos \beta}{2}\right)
$$

Then, the reflected irradiance from the ground [22] is:

$$
G_{r, \beta}=G H I \cdot \text { albedo } \cdot\left(\frac{1+\cos \beta}{2}\right)
$$

\section{2) PV output}

The efficiency of PV panels varies with the cell temperature which can be evaluated through [23].

$$
T_{c}=T_{a}+\frac{G_{g}}{G_{r e f}^{N O C T}} \cdot\left(\frac{9.5}{5.7+3.8 V_{w}}\right) \cdot\left(T_{c}^{N O C T}-T_{a}^{N O C T}\right) \cdot\left(1-\eta_{m}\right)(7)
$$

where $T_{a}$ is the ambient temperature, $G_{g, \beta}$ the global irradiance on the panel, $T_{c}^{N O C T}$ the Normal Operating Cell Temperature, $G_{r e f}^{N O C T}, T_{a}^{N O C T}$ the global irradiance and the ambient temperature in the NOCT conditions, $V_{w}$ the forecasted wind speed and $\eta_{m}$ the coefficient which summarizes the electrical efficiency and the heat transfer capability of PV modules.

Eq. 8 summarizes the influence on cell temperature on the PV output considering installation characteristics, weather conditions and cell parameters [23].

$$
P_{D C}=P_{i n s t} \frac{G_{g, \beta}}{G_{r e f}^{S T C}}\left[1-\gamma\left(T_{c}-T_{r e f}^{S T C}\right)+\mu \log _{10}\left(G_{g, \beta}\right)\right]
$$

Where $P_{D C}$ is the DC power delivered by the PV panels, $P_{\text {inst }}$ the rated power of the PV plant, $G_{g, \beta}$ the global irradiance on the PV panels, $G_{r e f}^{S T C}$ and $T_{r e f}^{S T C}$ the Standard Tests Conditions global irradiance and cell temperature, $T_{c}$ the cell temperature, $\gamma$ the power temperature coefficient of the cells, $\mu$ represents the effect of irradiance influence of the PV cell efficiency.

The inverter losses can be modelled with a quadratic function of the DC power.

$$
P_{l o s s}^{i n v}=a+b \cdot P_{D C}+c \cdot P_{D C}^{2}
$$

Then, the AC power delivered to the grid is done by

$$
P_{A C}=P_{D C}-P_{l o s s}^{i n v}
$$

\section{3) Parameter Optimisation and Forecasting}

To find the optimal parameters, an optimization procedure is run Evolutionary Particle Swarm Optimisation (EPSO) [24] which is a population-based method and relies on a set of moving solutions denoted as particles. In this case, a particle is a set of parameters to be applied to NWP in order to evaluate the AC PV outputs.

In this paper, the following parameters have been considered during the optimisation procedure:

- for installation/location characteristics: rated power, panel tilt angle, panel orientation azimuth and albedo

- $\quad$ for PV panel parameters: Normal Operating Cell Temperature, power temperature efficiency coefficient, irradiance efficiency coefficient, electrical efficiency and heat transfer coefficient, electrical efficiency and heat transfer coefficient

- inverter losses model coefficients.

The fitness function to be minimised is the mean absolute error (MAE) of the entire set of training data. The obtained parameters are then used to forecast the PV output from the testing data set NWP.

\section{Statistical Model}

The electrical-based method can take as inputs the different components of the GHI, as well as ambient temperature and wind speed. However, variables such as the cloudiness index cannot be directly included in the model. A statistical-based model that includes a larger set of explanatory variables was added to the forecasting system.

It is based on the component-wise gradient boosting (GB) technique, which is an ensemble machine-learning algorithm for regression [14]. It conducts numerical optimization, via steepest-descent, in function space by using a user-defined base learner recurrently on modified data that is the output from the previous iterations. Following the optimization phase, the final solution is a linear combination of the base learners.

The main feature of this algorithm is that the base learner selects only one predictor among all in each iteration and can includes different types of base learners, such as linear effects or smooth effects (e.g., P-splines). Therefore, this algorithm is capable of modelling non-linear relations and conducts feature selection.

The following additive model with $\mathrm{P}$-splines as base learners is fitted with the component-wise GB from $\mathrm{R}$ "mboost" package [25]:

$$
\begin{aligned}
& \hat{p}_{t+k}^{\prime n}=f\left(G_{g, t}\right)+f\left(T_{a, t}\right)+f\left(W_{m, t}\right)+f\left(C_{t, t}\right) \\
& +f\left(C_{h, t}\right)+f\left(C_{m, t}\right)+f\left(C_{l, t}\right)+f\left(R_{h, t}\right)+e_{t}
\end{aligned}
$$

where $f$ is a cubic smooth P-spline base-learner (function "bbs" in "mboost" package) with four degrees of freedom and 20 equally spaced knots. 


\section{Linear Pool}

The AR produces forecasts for the first lead-times with higher skill compared to the ones produced from NWP. Moreover, NWP with different parametrizations can be combined to improve the overall forecasting skill, as well as the electrical and statistical models. In order to produce a single forecast, the following linear pool was employed:

$$
\begin{aligned}
& \hat{p}_{t+k \mid t}^{n}=\beta_{0}+\beta_{1} \cdot p_{t+k \mid t}^{n, A R}+\beta_{2} \cdot p_{t+k \mid t}^{n, \text { elect }, N W P[1]}+\beta_{3} \cdot p_{t+k \mid t}^{n, G B, N W P[1]}+ \\
& \cdots+\beta_{m-1} \cdot p_{t+k \mid t}^{n, \text { elect }, N W P[z]}+\beta_{m-1} \cdot p_{t+k \mid t}^{n, G B, N W P[z]}+\varepsilon_{t+k \mid t}
\end{aligned}
$$

where $\beta$ are the model's coefficients and $z$ the number of different NWP models (in this paper, $z=2$ ).

A linear pool model is fitted for each lead-time with the RLS algorithm, which makes the model's coefficients timevarying.

\section{PROBABILISTIC ForECAST MODELS}

In order to produce probabilistic forecasts, a nonparametric approach based on local quantile regression is used [9][26]. The model for quantile $\tau$ consists in the following:

$$
\hat{p}_{t+k \mid t}^{\tau}=\omega\left(\hat{p}_{t+k \mid t}^{n}\right)+\varepsilon_{t+k \mid t}
$$

where $\omega($.$) is a coefficient function to be estimated and$ $\hat{p}_{t+k \mid t}^{n}$ is the normalized point forecast that includes information about the cloudiness level.

The $\omega($.$) function is estimated at a number of distinct$ reference points by using the kernel-local polynomial smoothing method, which means fitting a linear quantile regression model locally to each reference point. This allows the coefficients to vary smoothly according to the explanatory variables and therefore modelling nonlinear relations. A first order polynomial is considered and the tricube kernel with Euclidian norm is considered. An adaptive kernel bandwidth is defined by the nearest neighbour approach.

The function "rq" from R "quantreg" package [27] is used to solve the weighted quantile regression problem.

\section{RESUltS FROM THE DEMONSTRATION PHASE}

\section{A. Test Case Description}

The solar power dataset used as test case originates from a solar power plant located on the north of Portugal with 16 $\mathrm{kW}$ of nominal power.

For the meteorological dataset two NWP providers were used (NWP1 and NWP2). NWP1 does not provide forecasts for the DNI and DHI components, therefore the BolandRidley-Lauret model [28] is used to estimate their values.

This case study considers a dataset with hourly data (NWP and solar power) for the period between May 2013 and November 2014.

The point forecasts are evaluated with the mean absolute error (MAE) and root mean square error (RMSE) normalized by the rated power and calculated for each leadtime. Probabilistic forecasts are evaluated using the Continuous Ranked Probability Score (CRPS), described in [29].
A detailed list of the parameters and search intervals of the EPSO algorithm can be found in [13].

\section{B. Forecast Skill Results}

Fig. 2 presents the comparison, in terms of RMSE, of the electrical model considering as input the NWP from both providers considered independently and combined. Even though the model with NWP2 outperforms the one with NWP1 for all lead-times, the linear pool with both forecasts provides an increase of forecast skill, resulting in a smaller RMSE.

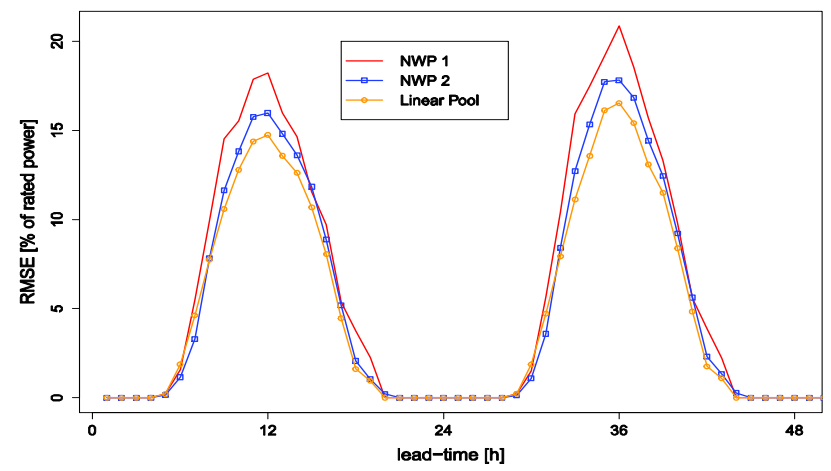

Figure 2. RMSE of the electrical model with NWP1, NWP2 and linear combination.

Fig. 3 depicts the RMSE for the electrical and statistical models separately and combined through the linear pool, using NWP2 as input. The statistical model outperforms the electrical model for all lead-times with the exception of the initial hours of the day where the electrical model shows better results (6h00 and $7 \mathrm{~h} 00)$. Even though the linear pool was not able to improve these first hours of the day, it shows a considerable decrease in the forecasting error for the rest of the day.

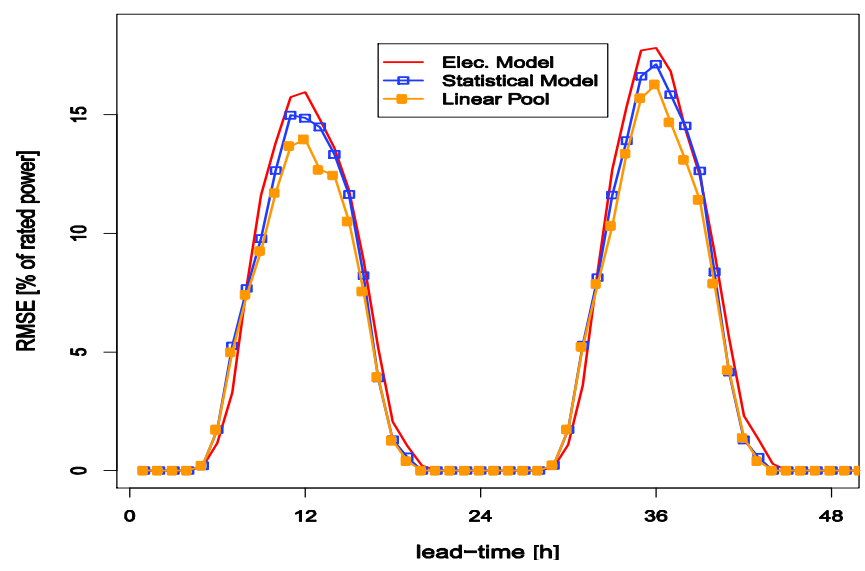

Figure 3. Comparison between electrical, statistical and linear pool models.

The forecasts used to obtain the errors presented in Fig.2 0and Fig. 3 were launched on a daily basis at midnight, hence the similarity between the solar cycle and the error curve.

From Fig. 4 onwards the predictions are updated on an hourly basis and integrates the forecasts generated by the AR model. Thus the error curve differs from the solar cycle. Note that all night-time hours were removed (filtered by the solar zenith angle).

Fig. 4 provides the final error of the forecasting system, which is obtained from the linear pool of all models: 
electrical model (with NWP1 and NWP2), statistical model (with NWP1 and NWP2) and AR model. The MAE ranges from $5.13 \%$ at the first lead-time to $10.39 \%$ for the last leadtime. The RMSE has its lower value at first lead-time with $8.21 \%$ and the $13.84 \%$ for the last one.

The lower values of error in the first lead-times are due the integration of the AR in the linear pool, which has been proved to outperform NWP models for very short-term horizon.

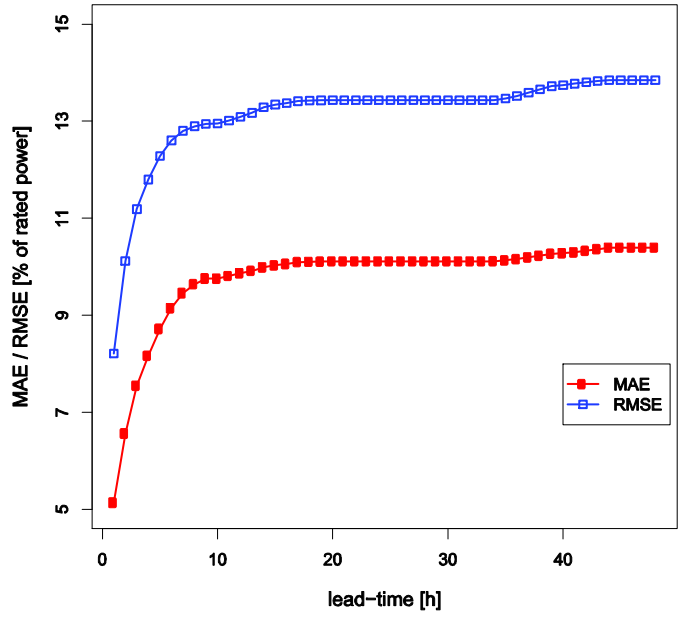

Figure 4. MAE and RMSE results of the forecasting system.

The performance of this hybrid forecasting tool was compared with two naïve models: persistence and diurnal model. The persistence model predicts the photovoltaic power for the next 48 hours by multiplying the last know value of normalized solar power by the clear sky model for the forecasting period. The diurnal model uses the last two days of measured solar power to predict 48 hours ahead.

The improvement over the naïve model, depicted in Fig.5 and Fig. 6, is obtained as follows:

$$
\text { Improvement }=\frac{\text { RMSE }(\text { Naive })-\text { RMSE }(\text { Hybrid })}{\text { RMSE }(\text { Naive })}
$$

For the first six lead-times, as illustrated in Fig. 5, the hybrid forecasting tool outperforms both naïve models. The smaller improvement occurs over the persistence model for the first lead-time with $9.55 \%$. However, five lead-times ahead the improved increases to $63.10 \%$, leading to the conclusion that the persistence model is only relevant for the first two lead-times.

Fig. 6 0illustrates the improvement towards the diurnal model considering the complete forecasting horizon. The persistence model is not represented in this figure, since its performance decreases abruptly for longer lead-times.

The higher improvement towards the diurnal model occurs at the first time-horizon reaching a value of $55.69 \%$ and the lower is at lead-time 20 with $28.07 \%$.

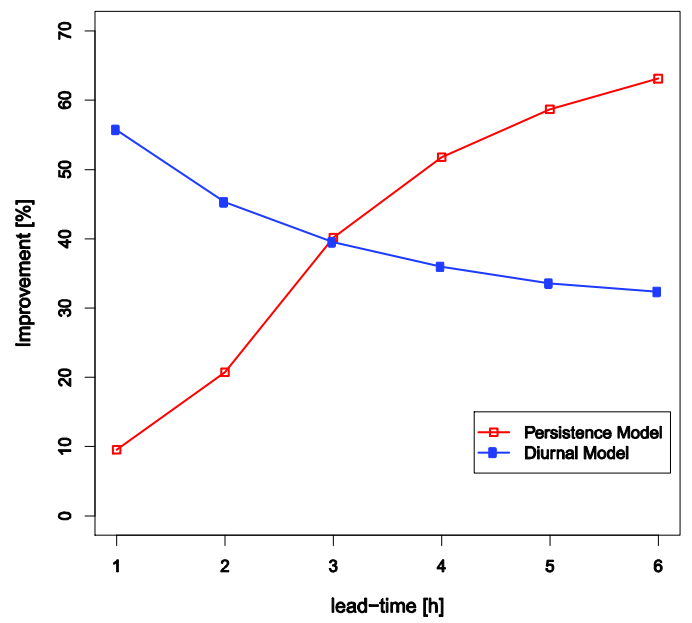

Figure 5. Point forecast improvement over naïve models.

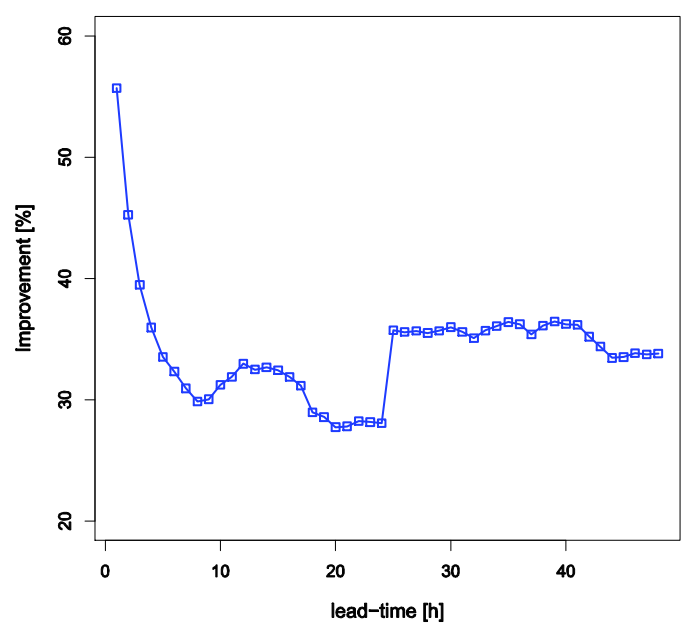

Figure 6. Point forecast improvement over diurnal model.

Fig. 7 0depicts the Continuous Ranked Probability Score (CRPS) calculated for each lead-time. It ranges between $3.35 \%$ and $5.17 \%$.

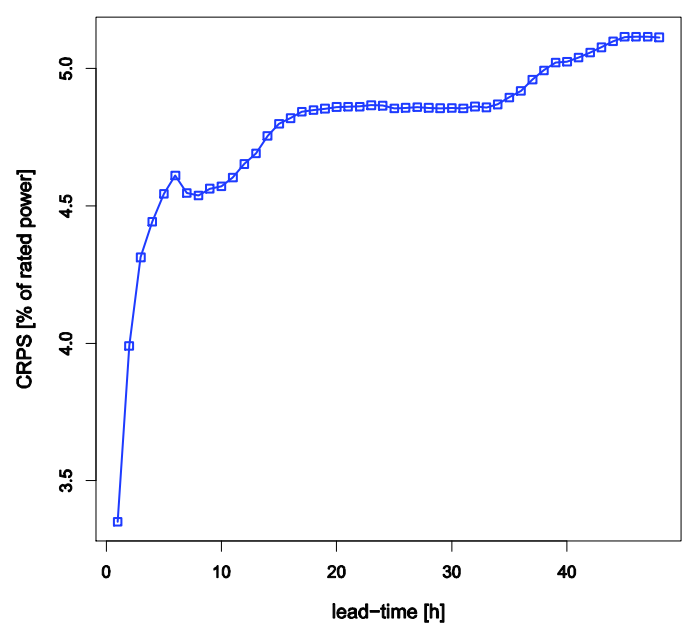

Figure 7. CRPS calculated for each lead-time. 


\section{CONCLUSIONS}

This paper describes a hybrid solar power forecasting system that explores the combination of physical and statistical models in order to improve the forecasting skill.

The operational demonstration for a PV installation in the north of Portugal showed the following main conclusions:

- Using multiple NWP services has shown relevant benefits, since the combination of forecasts from the same model but with different NWP inputs reveals better performance even when one of them is outperformed in all time-horizons;

- The combination of the electrical and statistical models has also demonstrated better performance considering that it results in a lower RMSE compared to the individual models run separately.

- Since the AR model is the most efficient model for very short-term horizon, its inclusion in the linear pool reduces the forecast error for the first leadtimes.

Furthermore, the average RMSE increases from 9.83\%, for the first 3 hours, to $13.37 \%$ for the rest of the forecasting period. Compared to two naïve models, the proposed hybrid forecasting tool shows an average improvement of $57.4 \%$ over persistence and $34.06 \%$ over the diurnal model.

The probabilistic forecast shows a good performance, with a CRPS ranging between $3.35 \%$ and $5.17 \%$ of the rated power.

\section{REFERENCES}

[1] M. Bazilian, I. Onyeji, M. Liebreich, I. MacGill, J. Chase, J. Shah, D. Gielen, D. Arent, D. Landfear, and S. Zhengrong, "Reconsidering the economics of photovoltaic power," Renewable Energy, vol. 53, pp. 329-338, May 2013.

[2] G. Masson, S. Orlandi, K. Petrick, V. Cassagne, J.I. Briano, M.J. Baez Morandi, R. Margolis, W. Rickerson, "PV development as prosumers: the role and challenges associated to producing and selfconsuming PV electricity," in Proc. of the 4th International Workshop on Integration of Solar Power into Power Systems, Berlin, Germany, Nov. 2014.

[3] M. A. Matos and R. J. Bessa, "Setting the operating reserve using probabilistic wind power forecasts," IEEE Transactions on Power Systems, vol. 26(2), pp.594-603, May 2011.

[4] A. Madureira, R.J. Bessa, J. Meirinhos, D. Fayzur, J. Filipe, A. Messias, D. Lopes. P. Matos, "The impact of solar power forecast errors on voltage control in smart distribution grids," in Proc. of the 23rd International Conference on Electricity Distribution - CIRED 2015, Lyon, France, June 2015.

[5] R.J. Bessa, C.L. Moreira, B. Silva, M.A. Matos, "Handling renewable energy variability and uncertainty in power systems operation," Wiley Interdisciplinary Reviews: Energy and Environment, vol. 3, no. 2, pp. 156-178, March/April 2014.

[6] L. A. Fernandez-Jimenez, A. Muñoz-Jimenez, A. Falces, M. Mendoza-Villena, E. Garcia-Garrido, P.M. Lara-Santillan, E. Zorzano-Alba, and P.J. Zorzano-Santamaria, "Short-term power forecasting system for photovoltaic plants," Renewable Energy, vol. 44, pp. 311-317, Aug. 2012.

[7] M. Zamo, O. Mestre, P. Arbogast, and O. Pannekoucke, "A benchmark of statistical regression methods for short-term forecasting of photovoltaic electricity production, part I: Deterministic forecast of hourly production," Solar Energy, vol. 105, pp. 792-803, July 2014.
[8] C. Chen, S. Duan, T. Cai, and B. Liu, "Online 24-h solar power forecasting based on weather type classification using artificial neural network," Solar Energy, vol. 85, pp. 2856-2870, Nov. 2011.

[9] P. Bacher, H. Madsen, and H.A. Nielsen, "Online short-term solar power forecasting," Solar Energy, vol. 83, no. 10, pp. 1772-1783, 2009.

[10] H. Pedro and C. Coimbra, "Assessment of Forecasting Techniques for Solar Power Production with no Exogenous inputs," Solar Energy, vol. 86, no. 7, pp. 2017-2028, Jul. 2012.

[11] R.J. Bessa, A. Trindade, and V. Miranda, "Spatial-temporal solar power forecasting for smart grids," IEEE Transactions on Industrial Informatics, vol. 11, no. 1, pp. 232-241, Feb. 2015.

[12] R. H. Inman, H. Pedro, and C. Coimbra, "Solar forecasting methods for renewable energy integration," Progress in Energy and Combustion Science, vol. 39, no. 6, pp. 535-576, Dec. 2013.

[13] J. Sumaili, R.J. Bessa, F. Rahman, R. Tomé, J.N. Sousa, "Electrical model parameter characterization for short-term solar power forecasting," in Proc. of the 4th International Workshop on Integration of Solar Power into Power Systems, Berlin, Germany, Nov. 2014.

[14] P. Bühlmann, "Boosting for high-dimensional linear models," Ann. of Stat., vol. 34, no. 2, pp. 559-583, 2006.

[15] W.C. Skamarock, et al., A description of the advanced research WRF version 3. NCAR tech. note TN-475_STR, 2008.

[16] E.S. Mlawer, S. Taubman, P. Brown, M. Iacono, and S. Clough, "Radiative transfer for inhomogeneous atmosphere: RRTM, a validated correlated-k model for the long wave," J. Geophys. Res., vol. 102, 16663-16682, 1997.

[17] H. Madsen, Time Series Analysis, London: Chapman and Hall, 2006.

[18] G. Blaesser and D. Munro, "Guidelines for the assessment of photovoltaic plants - initial and periodic tests on PV plants," European Commission, Joint Research Centre, Ispra, Italy, 1995.

[19] E. Lorenz, J. Hurka, D. Heinemann, and H.G. Beyer "Irradiance forecasting for the power prediction of grid-connected photovoltaic systems," IEEE Journal of Selected Topics in Applied Earth Observations and Remote Sensing, vol. 2(1), pp. 2-10, Mar. 2009.

[20] D.L. King, J.A. Kratochvil, W.E. Boyson. "Spectral and angle-ofincidence effects on photovoltaic modules and solar irradiance sensors," in Proc. Of the 26th IEEE Photovoltaic Specialists Conference, Sept. 1997.

[21] PV_LIB TOOLBOX, http://pvpmc.org/pv-lib/functions-bycatagory/pvl_kingdiffuse/ (accessed on September 12, 2014)

[22] P.G. Loutzenhiser, H. Manz, C. Felsmann, P.A. Strachan, T. Frank, and G.M. Maxwell, "Empirical validation of models to compute solar irradiance on inclined surfaces for building energy simulation," Solar Energy vol. 81. pp. 254-267, 2007.

[23] C. Cristofari, P. Poggi, G. Notton, and M. Muselli, "Thermal modeling of a photovoltaic module," in Proc. of the $6^{\text {th }}$ International Conference on Simulation and Optimization (IASTED 2006), pp. 273-278, Gaborone, Botswana, September 2006.

[24] V. Miranda, H. Keko, and A.J. Duque, "EPSO: Evolutionary particle swarms," in Advances in Evolutionary Computing for System Design, ser. Studies in Computational Intelligence, L. Jain, Ed. et al. New York: Springer, 2007, vol. 66, ch. 6, pp. 139-168.

[25] B. Hofner, A. Mayr, N. Robinzonov, M. Schmid (2014). "Modelbased Boosting in R: A Hands-on Tutorial Using the R Package mboost," Computational Statistics, vol. 29, pp. 3-35, 2014.

[26] J. Fan and W. Zhang, "Statistical methods with varying coefficient models," Statistics and Its Interface, vol. 1, pp. 179-195, 2008.

[27] R. Koenker, quantreg: Quantile Regression. R package version 5.05, 2013. http://CRAN.R-project.org/package= quantreg

[28] B. Ridley, J. Boland, P. Lauret, "Modelling of diffuse solar fraction with multiple predictors," Renewable Energy, vol. 35(2), pp. 478483, Feb. 2010.

[29] G. Anastasiades and P. McSharry, "Quantile forecasting of wind power using variability indices," Energies, vol. 6, no. 2, pp. 662-695, 2013. 